\title{
Evaluation of Molecular Orientation in Aromatic Polyimide Films by FT-IR Reflection Absorption Spectroscopy
}

\author{
Kazuo Iida, Yukihiro Imamura, Can LiaO, \\ Shuhei NaKAMURA, and Goro SaWA \\ Department of Electrical and Electronic Engineering, Mie University, Tsu 514, Japan
}

(Received September 25, 1995)

\begin{abstract}
A method for the quantitative evaluation of molecular orientation in organic film containing rigid parts has been developed. In the method, mean square of cosine of angle between normal to the substrate and transition dipole moment vectors due to molecular vibration was evaluated by means of comparing infrared reflection-absorption (RA) spectrum of a film with the transmission spectrum of the same molecule in the unoriented bulk state such as in $\mathrm{KBr}$ pellets. Application of the method to the polyimide films prepared by vapor deposition polymerization showed that both the molecular chain axis and zigzag plane of the chain were nearly parallel to the substrate, and that the pyromellitimide and the phenylene ring were inclined to the substrate. As the heating temperature for the imidization rose, both the chain axis and the zigzag plane showed a tendency to be parallel to the substrate, the phenylene ring showed a tendency to cline, but the orientation of the pyromellitimide did not vary. The various factors that influence the accuracy of the orientation analysis were also discussed.

KEY WORDS Molecular Orientation / Polyimide / Infrared Spectroscopy / Reflection Absorption Spectroscopy
\end{abstract}

In recent years, much attention has been attracted to aromatic polyimide (PI) films, which have been used in a wide variety of applications. Concomitantly, there has been an increased need for the establishment of a characterization method. Experimental characterization of the molecular orientation is fundamental to understanding the properties of polymeric films. Common methods for quantifying molecular orientation include infrared (IR) dichroism, birefringence, polarized Raman spectroscopy, polarized fluorescence spectroscopy, X-ray scattering, and NMR spectroscopy. ${ }^{1-3}$ Among the various analytical methods applicable to thin organic films, infrared spectroscopy provides the most convenient and useful way for the analysis of constituent groups in a molecule. Several attempts have also been made to apply IR reflection-absorption (RA) spectroscopy for this purpose. ${ }^{4,5}$ For example, Allara and Nuzzo ${ }^{4}$ have shown that the orientation of transition moments for various vibration modes of molecules in LB films can be determined by comparing the RA spectrum of a monolayer film with the transmission spectrum of the same molecule in the unoriented bulk state such as in $\mathrm{KBr}$ pellet. Umemura, et al. ${ }^{5}$ have shown that the orientation of molecules in LB films can be determined by comparing the RA spectrum of LB film on a metal surface with the transmission spectrum of LB film on a transparent substrate such as $\mathrm{ZnSe}$ and $\mathrm{CaF}_{2}$. Although their methods are, in principle, reasonable, it is not necessarily convenient for practical purposes, requiring the optical parameters of the sample or the substrate. In their method, angles between a transition moment vector and the electric vectors of infrared light has been discussed. On the contrary, we expect that angles between an electric vector of infrared light and three transition moment vectors perpendicular to each other originated from molecular orientations of rigid parts such as phenylene rings can be determined from a spectrum by a polarized light.

The morphology of PI in the solid state has been investigated by numerous groups using scattering and diffraction techniques. ${ }^{6-9}$ From a review of the literature, it is concluded that the molecule has a planar zigzag conformation, and that the molecular orientation is parallel to the substrate. Russell et al. ${ }^{10}$ noted that the use of the term crystalline is questionable. Takahashi et $a l .{ }^{8}$ described the structure of PI in the bulk in term of a "smectic" ordering. The molecule of PI has rigid pyromellitimide and oxyphenylene in the main chain. Thus, the molecular orientation of PI can be determined by the direction of the chain axis, rotation angle of the plane of the zigzag chain on the chain axis, and rotation angle of the pyromellitimide and the phenylene ring against the axis of the segment of zigzag chain. Though X-ray analysis can be used to show a planar zigzag conformation of the chain and the molecular orientation of the chain to be parallel to the substrate, it can not show the orientation of the plane of the zigzag chain and the pyromellitimide and the phenylene ring. Recently, Perez et al. referred to the orientation of the imide rings on a basis of two IR absorption intensities due to symmetric and asymmetric $\mathrm{C}=\mathrm{O}$ stretching vibrations in $\mathrm{RA}$ spectra. ${ }^{11}$ Their manner is lacking in generality for estimation of molecular orientation, because it is valid only when PI chains orient parallel to the substrate.

In the present paper, we propose a method to evaluate to the orientation of organic molecules containing rigid parts. We apply it to characterize the molecular orientation in PI films prepared by vapor deposition polymerization (VDP) from pyromellitic dianhydride (PMDA) and diaminodipheny ether (DDE). The reason why we adopted PI film is that they are a typical heat resistant polymer and have an abundance of structural study, but the molecular orientation besides the molecular chain axis is not clear. The other reason is that the spectra of the films on the evaporated gold, which is employed for evaluation of electric properties, can be obtained by RA spectroscopy. 


\section{EXPERIMENTAL}

The polyimide films were prepared by VDP on an evaporated gold on glass substrate, as described elsewhere. ${ }^{12}$ The VDP system contains two evaporation sources: one for PMDA and the other for DDE. The evaporation rate could be adjusted separately by a power source with crystal oscillator rate monitor so as to maintain a stoichiometric ratio. The substrate was heated to about $70^{\circ} \mathrm{C}$ for reevaporation of nonreacted monomers. For imidization, subsequent heating was performed at 200 to $350^{\circ} \mathrm{C}$ for $1 \mathrm{~h}$ in vacuum after removal from the deposition system.

IR spectra were obtained using a Jasco FT/IR 7000 Fourier transform IR spectrometer equipped with a DTGS detector with a resolution $4 \mathrm{~cm}^{-1}$. For the RA measurements of films on gold, a Jasco PR-500 reflection attachment was used at the angle of incidence of $85^{\circ}$ using $p$-polarized radiation by a wire-grid polarizer. Spectra of unoriented sample were obtained using normal incidence transmission spectra of pressed $\mathrm{KBr}$ disks. The number of interferogram accumulation was 256 scans for both RA and transmission measurements.

\section{EVALUATION OF MOLECULAR ORIENTATION}

A direction of orientation of molecules is so far usually determined from three spectra with different directions of incident lights. ${ }^{4,5,13}$ In these method, angles between a direction of a transition dipole moment and three directions of electric field components of incident lights are determined. From the opposite viewpoint, angles between a direction of a field component and directions of three transition dipole moments perpendicular to each other ought to be determined. Since molecular vibrations of a rigid unit usually have many transition moments with different directions, there are surely three transition moments perpendicular to each other.

The IR absorption intensity $A_{i}$ of the $i$ th vibration mode is proportional to the mean square of inner product of the transition moment $\mu$ and field component $\mathrm{E}$ of IR light.

$$
A_{i} \propto\left\langle\left|\mu_{i} \cdot E\right|^{2}\right\rangle
$$

Ratios of peak intensities, $A_{i}, A_{j}$, and $A_{k}$, due to the molecular orientation with transition moments perpendicular to each other are described by following equation:

$$
\begin{aligned}
& A_{i}: A_{j}: A_{k} \\
& \quad=\mu_{i}^{2} E^{2}\left\langle\cos ^{2} \theta_{i}\right\rangle: \mu_{j}^{2} E^{2}\left\langle\cos ^{2} \theta_{j}\right\rangle: \mu_{k}^{2} E^{2}\left\langle\cos ^{2} \theta_{k}\right\rangle
\end{aligned}
$$

where $\theta$ is the angle between $\mu$ and $E$. Since $\cos \theta$ is the direction cosine in the rectangular coordinates,

$$
\left\langle\cos ^{2} \theta_{i}\right\rangle+\left\langle\cos ^{2} \theta_{j}\right\rangle+\left\langle\cos ^{2} \theta_{k}\right\rangle=1
$$

For organic molecules in the unoriented bulk state such as in $\mathrm{KBr}$ pellets,

$$
\left\langle\cos ^{2} \theta_{i}\right\rangle=\left\langle\cos ^{2} \theta_{j}\right\rangle=\left\langle\cos ^{2} \theta_{k}\right\rangle=\frac{1}{3}
$$

Thus, ratios of peak intensities, $A_{i}^{\mathrm{KBr}}, A_{j}^{\mathrm{KBr}}$, and $A_{k}^{\mathrm{KBr}}$, are expressed as follows

$$
A_{i}^{\mathrm{KBr}}: A_{j}^{\mathrm{KBr}}: A_{k}^{\mathrm{KBr}}=\mu_{i}^{2}: \mu_{j}^{2}: \mu_{k}^{2}
$$

This equation means that the ratios of the peak intensities in the spectrum of $\mathrm{KBr}$ pellets correspond to the ratios of the square of the transition dipole moments. Since there is only a perpendicular field component at the surface in RA spectroscopy, only molecules with a finite transition moment component perpendicular to the substrate are observable. Ratios of peak intensities, $A_{i}^{\text {RAS }}$, $A_{j}^{\mathrm{RAS}}$, and $A_{k}^{\mathrm{RAS}}$, in RA spectrum are given by

$$
\begin{aligned}
& A_{i}^{\mathrm{RAS}}: A_{j}^{\mathrm{RAS}}: A_{k}^{\mathrm{RAS}} \\
& \quad=A_{i}^{\mathrm{KBr}}\left\langle\cos ^{2} \theta_{i}\right\rangle: A_{j}^{\mathrm{KBr}}\left\langle\cos ^{2} \theta_{j}\right\rangle: A_{k}^{\mathrm{KBr}}\left\langle\cos ^{2} \theta_{k}\right\rangle
\end{aligned}
$$

Therefore, $\left\langle\cos ^{2} \theta_{i}\right\rangle,\left\langle\cos ^{2} \theta_{j}\right\rangle$, and $\left\langle\cos ^{2} \theta_{k}\right\rangle$, can be determined from eq 3 and 6 as follows:

$$
\begin{aligned}
& \left\langle\cos ^{2} \theta_{i}\right\rangle \\
& =\frac{A_{i}^{\mathrm{RAS}} A_{j}^{\mathrm{KBr}} A_{k}^{\mathrm{KBr}}}{A_{i}^{\mathrm{RAS}} A_{j}^{\mathrm{KBr}} A_{k}^{\mathrm{KBr}}+A_{i}^{\mathrm{KBr}} A_{j}^{\mathrm{RAS}} A_{k}^{\mathrm{KBr}}+A_{i}^{\mathrm{KBr}} A_{j}^{\mathrm{KBr}} A_{k}^{\mathrm{RAS}}} \\
& \left\langle\cos ^{2} \theta_{j}\right\rangle \\
& =\frac{A_{i}^{\mathrm{KBr}} A_{j}^{\mathrm{RAS}} A_{k}^{\mathrm{KBr}}}{A_{i}^{\mathrm{RAS}} A_{j}^{\mathrm{KBr}} A_{k}^{\mathrm{KBr}}+A_{i}^{\mathrm{KBr}} A_{j}^{\mathrm{RAS}} A_{k}^{\mathrm{KBr}}+A_{i}^{\mathrm{KBr}} A_{j}^{\mathrm{KBr}} A_{k}^{\mathrm{RAS}}} \\
& \left\langle\cos ^{2} \theta_{k}\right\rangle \\
& =\frac{A_{i}^{\mathrm{KBr}} A_{j}^{\mathrm{KBr}} A_{k}^{\mathrm{RAS}}}{A_{i}^{\mathrm{RAS}} A_{j}^{\mathrm{KBr}} A_{k}^{\mathrm{KBr}}+A_{i}^{\mathrm{KBr}} A_{j}^{\mathrm{RAS}} A_{k}^{\mathrm{KBr}}+A_{i}^{\mathrm{KBr}} A_{j}^{\mathrm{KBr}} A_{k}^{\mathrm{RAS}}}
\end{aligned}
$$

Equations 7-9 mean that the molecular orientation of organic compound on metallic substrate can be quantitatively evaluated from FT-IR RA spectrum and the transmission spectrum of the same molecule in the unoriented bulk state such as in $\mathrm{KBr}$ pellets. Three transition dipole moments are not necessary to be perpendicular to each other, but, if so, $\left\langle\cos ^{2} \theta\right\rangle$ should be represented by means of a more complicated expression than eq 7-9. Fortunately, rigid parts may usually have three vibration modes with transition dipole moments perpendicular to each other.

\section{RESULTS AND DISCUSSION}

Figure 1 shows the transmission spectrum of $\mathrm{PI}$ in $\mathrm{KBr}$ pellet and the RA spectrum of PI on the evaporated gold. PI was prepared by VDP and subsequent heating at $300^{\circ} \mathrm{C}$. These spectra show almost the same bands, but the relative intensities of the peaks are distinctly different. Since the PI molecules in $\mathrm{KBr}$ pellet are randomly oriented, difference in relative intensities of the peaks in the transmission and RA spectra indicates the existence of the molecular orientation. The wavenumbers of absorption peaks, vibrational mode assignments ${ }^{14}$ and the direction of the transmission dipole moment are given in Table I. The chemical structure of PI is shown in Figure 2 together with the directions of the transition moments. Since the complicated molecular structure makes the assignments of vibrational modes very difficult, some assignments listed in Table I are presumed. Though there is no objection to the assignments of main peaks 
at $1780,1720,1500,1380,1250$, and $725 \mathrm{~cm}^{-1}$, the assignments of minor peaks at $1170,1015,880$, and 820 $\mathrm{cm}^{-1}$ are not consistent between some literature. ${ }^{14-16}$

Ishida et al. described that the $\mathrm{C}=\mathrm{O}$ asymmetric stretching mode of PI changed from 1717 to $1725 \mathrm{~cm}^{-1}$ by crystallization. ${ }^{14}$ It is reported that the $\mathrm{C}=\mathrm{O}$ stretching peak of PI by $\mathrm{VDP}^{16,17}$ and very thin $\mathrm{PI}^{11}$ appears at $1730^{16}$ and $1738^{11,17} \mathrm{~cm}^{-1}$. Thus, the wave-

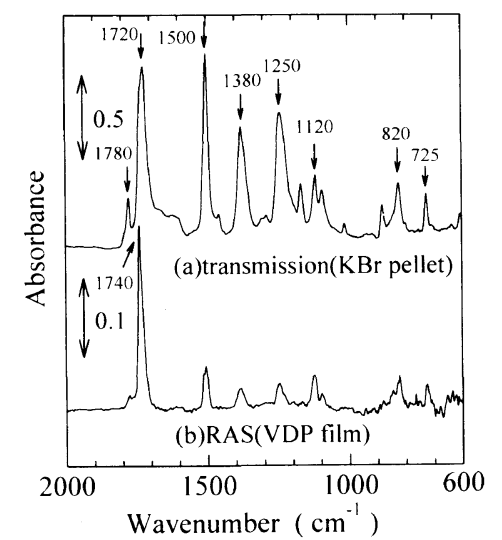

Figure 1. Transmission and RA spectra of polyimide.

Table I. Absorption peaks of polyimide

\begin{tabular}{|c|c|c|c|}
\hline $\mathrm{KBr}$ & RAS & \multirow{2}{*}{ Assignments } & \multirow{2}{*}{$\begin{array}{l}\text { Direction } \\
\text { of moment }\end{array}$} \\
\hline $\mathrm{cm}^{-1}$ & $\mathrm{~cm}^{-1}$ & & \\
\hline 1777 & 1777 & $\mathrm{C}=\mathrm{O}$ symmetric stretching & B \\
\hline 1725 & 1742 & $\mathrm{C}=\mathrm{O}$ asymmetric stretching & $\mathrm{C}$ \\
\hline 1502 & 1506 & $\mathrm{C}=\mathrm{C}$ stretching $(\mathrm{DDE})$ & \\
\hline 1379 & 1383 & $\mathrm{~N}-\mathrm{C}$ stretching & B \\
\hline 1241 & 1249 & $\mathrm{Ph}-\mathrm{O}-\mathrm{Ph}$ asymmetric stretching & A \\
\hline 1168 & 1170 & & \\
\hline 1118 & 1123 & $\mathrm{C}-\mathrm{H}$ in-plane bending (DDE) & $\mathrm{C}$ \\
\hline 1094 & 1098 & & \\
\hline 1015 & $(1015)^{\mathrm{b}}$ & & \\
\hline 882 & $(880)^{\mathrm{b}}$ & C-H out-of-plane bending & $\mathrm{D}$ \\
\hline 824 & 832 & (DDE) & \\
\hline 725 & 727 & $\mathrm{C}=\mathrm{O}$ out of plane bending & $\mathrm{D}$ \\
\hline
\end{tabular}

${ }^{a}$ A, parallel to polymer chain axis; B, parallel to segment of zigzag chain; C, normal to segment of zigzag and in plane of rigid unit; D, normal to segment of zigzag and out of plane of rigid unit.

${ }^{b}(\cdots)$ : very weak peak.

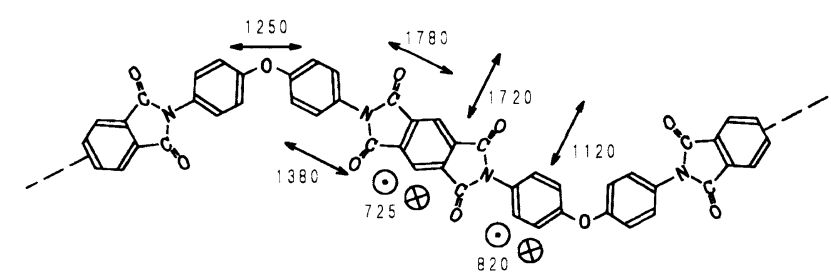

Figure 2. Chemical structure of PI together with the directions of the transition moments and wavenumbers of the seven molecular vibrations. number of $1742 \mathrm{~cm}^{-1}$ may present for the crystallization of PI by VDP.

The direction of the transition dipole moment of $\mathrm{Ph}$ $\mathrm{O}-\mathrm{Ph}$ asymmetric stretching at $1250 \mathrm{~cm}^{-1}$ is parallel to the axis of the polymer chain. The directions of the transition dipole moments of $\mathrm{C}=\mathrm{O}$ symmetric stretching at $1780 \mathrm{~cm}^{-1}$ and $\mathrm{C}-\mathrm{N}$ stretching at $1380 \mathrm{~cm}^{-1}$ are parallel to the segment of zigzag chain. If two phenylene groups in diphenyl ether portions couple with each other, the direction of the transition dipole moments of $\mathrm{C}=\mathrm{C}$ stretching at $1500 \mathrm{~cm}^{-1}$ will be parallel to the axis of the polymer chain, and if not, it will be parallel to the segment. The directions of the transition dipole moments of $\mathrm{C}-\mathrm{H}$ in-plane bending at $1120 \mathrm{~cm}^{-1}$ and $\mathrm{C}-\mathrm{H}$ outof-plane bending at $820 \mathrm{~cm}^{-1}$ are perpendicular to each other and these two directions are perpendicular to the segments. Thus, when the peak at 1780 or $1380 \mathrm{~cm}^{-1}$ is added to two peaks at 1120 and $820 \mathrm{~cm}^{-1}$, the orientation of the phenylene rings of DDE can be determined according to eqs $7-9$. As the peak intensity at $1380 \mathrm{~cm}^{-1}$ is larger than that at $1780 \mathrm{~cm}^{-1}$, it has less error factors Therefore, we use three peaks at $1380,1120,820 \mathrm{~cm}^{-1}$ to determine the orientation of the phenylene rings. In the same manner, the orientation of the pyromellitimide is determined using three peak intensities of $\mathrm{C}=\mathrm{O}$ asymmetric stretching at $1720 \mathrm{~cm}^{-1}, \mathrm{C}=\mathrm{O}$ out-of-plane bending at $725 \mathrm{~cm}^{-1}$, and $\mathrm{C}-\mathrm{N}$ stretching at $1380 \mathrm{~cm}^{-1}$. The orientation of the axis is determined from the ratio of the peak intensity at $1250 \mathrm{~cm}^{-1}$ to one peak intensity of above peaks. For example, if $\left\langle\cos ^{2} \theta_{1720}\right\rangle$ is already known, $\left\langle\cos ^{2} \theta_{1250}\right\rangle$ will be determined from following equation:

$$
A_{1720}^{\mathrm{RAS}}: A_{1250}^{\mathrm{RAS}}=A_{1720}^{\mathrm{KBr}}\left\langle\cos ^{2} \theta_{1720}\right\rangle: A_{1250}^{\mathrm{KBr}}\left\langle\cos ^{2} \theta_{1250}\right\rangle .
$$

Table II shows values of $\left\langle\cos ^{2} \theta\right\rangle$ of the peaks. Two $\left\langle\cos ^{2} \theta_{1380}\right\rangle$ ought to have the same value in principle. The disagreement between two $\left\langle\cos ^{2} \theta_{1380}\right\rangle$ in Table II may be caused by disturbance by noises at wavenumbers blow $1000 \mathrm{~cm}^{-1}$ in RA spectrum, effect of neighbor peak on the base line and imperfect molecular structure, but it is not too large.

$\left\langle\cos ^{2} \theta_{1250}\right\rangle$ smaller than $1 / 3$ means that chain orientation of PI is roughly parallel to the substrate. The orientation of the plane of the zigzag chain can be determined by a direction of the chain axis and tilt angle around the chain axis. Segments of the planar zigzag chain make an angle of 126 degrees, that is, the segments and the chain axis intersect at an angle of 27 degrees. The transition moments of vibrations at 1380 and $1250 \mathrm{~cm}^{-1}$ are in the same plane and these at $1380 \mathrm{~cm}^{-1}$ make an angle of 27 degrees to that at $1250 \mathrm{~cm}^{-1}$. When the chain axis at $\alpha$ degrees to the substrate and the zigzag plane turn at $\beta$ degrees around the axis, the segments

Table II. Orientation of polyimide

\begin{tabular}{|c|c|c|c|c|c|c|}
\hline \multicolumn{3}{|c|}{ Pyromellitimide } & \multicolumn{3}{|c|}{ Phenylene ring } & \multirow{2}{*}{$\begin{array}{l}\text { Chain axis } \\
\left\langle\cos ^{2} \theta_{1250}\right\rangle\end{array}$} \\
\hline$\left\langle\cos ^{2} \theta_{1380}\right\rangle$ & $\left\langle\cos ^{2} \theta_{1720}\right\rangle$ & $\left\langle\cos ^{2} \theta_{725}\right\rangle$ & $\left\langle\cos ^{2} \theta_{1380}\right\rangle$ & $\left\langle\cos ^{2} \theta_{1120}\right\rangle$ & $\left\langle\cos ^{2} \theta_{820}\right\rangle$ & \\
\hline 0.10 & 0.57 & 0.33 & 0.15 & 0.41 & 0.44 & 0.10 \\
\hline
\end{tabular}


next to each other have different tilt angle $\chi_{1}$ and $\chi_{2}$ to the substrate, described by following equations

$$
\begin{aligned}
& \sin \chi_{1}=\sin 27 \sin \alpha+\sin 27 \cos \alpha \sin \beta \\
& \sin \chi_{2}=\sin 27 \sin \alpha-\sin 27 \cos \alpha \sin \beta
\end{aligned}
$$

If this configuration of the PI chain describes the average orientation of PI chain, the mean square of cosine of the angle of the segment from the substrate normal, $\left\langle\cos ^{2} \theta_{1380}\right\rangle$ can be expressed by $\alpha$ and $\beta$ as following equation.

$$
\begin{aligned}
\left\langle\cos ^{2} \theta_{1380}\right\rangle & =\frac{1}{2}\left\{\left\langle\cos ^{2}\left(90-\chi_{1}\right)\right\rangle+\left\langle\cos ^{2}\left(90-\chi_{2}\right)\right\rangle\right\} \\
& =\cos ^{2} 27\left\langle\sin ^{2} \alpha\right\rangle+\sin ^{2} 27\left\langle\cos ^{2} \alpha \sin ^{2} \beta\right\rangle
\end{aligned}
$$

From $\left\langle\cos ^{2} \theta_{1250}\right\rangle=\left\langle\sin ^{2} \alpha\right\rangle$ and approximation of $\left\langle\cos ^{2} \alpha \sin ^{2} \beta\right\rangle$ to $\left\langle\cos ^{2} \alpha\right\rangle\left\langle\sin ^{2} \beta\right\rangle,\left\langle\sin ^{2} \beta\right\rangle$ can be obtained. If the both distributions of $\cos ^{2} \alpha$ and $\sin ^{2} \beta$ are narrow, an error of the approximation will be small. $\left\langle\sin ^{2} \beta\right\rangle$ is estimated at 0.24 from the average of two $\left\langle\cos ^{2} \theta_{1380}\right\rangle$ and $\left\langle\cos ^{2} \theta_{1250}\right\rangle$ listed in Table II. It means that the plane of the zigzag chain is nearly parallel to the substrate. Large value of $\left\langle\cos ^{2} \theta_{1720}\right\rangle$ shows a large tilt of the pyromellitimide.

Figure 3 shows the RA spectra of PI film which was heated at a temperature from 200 to $350^{\circ} \mathrm{C}$. The heating temperature was varied at intervals of 50 degrees. As the heating temperature is increased, the peak intensities at $1780,1500,1380$, and $1250 \mathrm{~cm}^{-1}$ decrease, but those at 1740 and $1120 \mathrm{~cm}^{-1}$ slightly increase. These results indicate that the molecular orientation varies with the heating temperature. Figure 4 shows the variation of $\left\langle\cos ^{2} \theta\right\rangle$ with the heating temperature. $\left\langle\cos ^{2} \theta_{1380}\right\rangle$ and $\left\langle\cos ^{2} \theta_{1250}\right\rangle$ decrease with an increase in the heating temperature, but instead $\left\langle\cos ^{2} \theta_{1720}\right\rangle$ and $\left\langle\cos ^{2} \theta_{1120}\right\rangle$ increase, and $\left\langle\cos ^{2} \theta_{820}\right\rangle$ and $\left\langle\cos ^{2} \theta_{725}\right\rangle$ do not change remarkably.

The decrease of $\left\langle\cos ^{2} \theta_{1380}\right\rangle$ for the pyromellitimide, little variation of $\left\langle\cos ^{2} \theta_{725}\right\rangle$ and the increase of $\left\langle\cos ^{2} \theta_{1720}\right\rangle$ with the heating temperature imply that the angle of inclination of the segment decreases and the angle of the pyromellitimide around the segment increases as the heating temperature rises. The decrease of $\left\langle\cos ^{2} \theta_{1380}\right\rangle$ for the phenylene ring, the increase of $\left\langle\cos ^{2} \theta_{1120}\right\rangle$ and little variation of $\left\langle\cos ^{2} \theta_{820}\right\rangle$ imply that the angle inclination of the segment decreases and that the angle of the phenylene ring around the segment increases, but is smaller than that of the pyromellitimide.

The above variation of the molecular orientation with the heating temperature is the average of the orientation. PI contains amorphous phase and ordered phase, which is intermediate between crystalline and amorphous phases. $^{7,8}$ The molecules in the ordered phase orient parallel to the substrate. ${ }^{6-9}$ The variation may be caused by either the further orientation of the molecule in the ordered phase or the increase in the region of the ordered phase. X-Ray diffraction analysis shows that the molecules do not orient on the tilt to the substrate but parallel. Therefore, the variation is caused by the increase in the region of the ordered phase. If a specimen is homogeneous such as LB film, $\left\langle\cos ^{2} \theta\right\rangle$ will give a

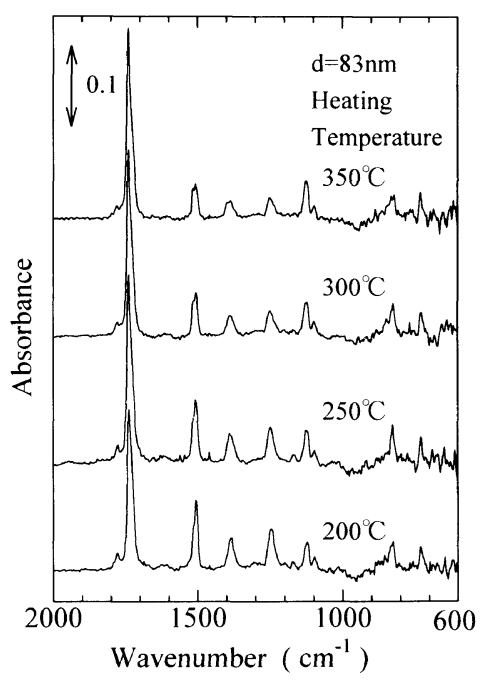

Figure 3. Variation of RA spectra with heating temperature.

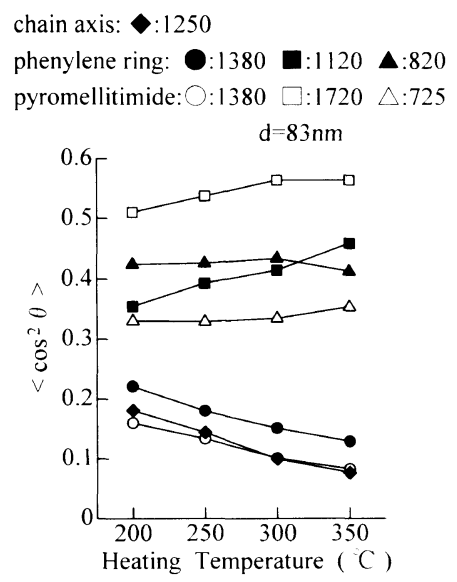

Figure 4. Variation of molecular orientation with heating temperature.

concrete angle of the molecular orientation. However, when a specimen is heterogeneous, $\left\langle\cos ^{2} \theta\right\rangle$ only gives the measure of the molecular orientation.

Now let us discuss two problems to be noted when we use this evaluation method. First, we have to consider the sample thickness. We described that there is only a perpendicular field component in RA measurements. This is valid only if the film thickness is sufficiently small as compared with the IR wavelength. A component due to electric field component parallel to the substrate in a peak intensity increases with increasing thickness and decreases with increasing wavelength. The shortest wavelength of peak used in above estimation, $5.8 \mu \mathrm{m}$, corresponding to $1740 \mathrm{~cm}^{-1}$, is about 70 times larger than the sample thickness, $0.083 \mu \mathrm{m}$. The longest wavelength is $13.8 \mu \mathrm{m}$, corresponding to $725 \mathrm{~cm}^{-1}$. The absorption peak intensity $A_{1740}^{\text {RAS }}$ at $1740 \mathrm{~cm}^{-1}$ contains larger error factor than $A_{725}^{\mathrm{RAS}}$. As the denominator and numerator in eq 7-9 are linear expression of $A^{\mathrm{RAS}}$, the error factor in $A^{\mathrm{RAS}}$ at the longest wavelength is canceled. Therefore, the error factor in $\left\langle\cos ^{2} \theta\right\rangle$ becomes smaller than the error factor in $A^{\mathrm{RAS}}$ at the shortest wavelength.

The other problem is how the peak intensity is evaluated. The peak intensity is evaluated from peak height in this paper. The peak of $\mathrm{C}=\mathrm{O}$ asymmetric stretching mode at $1740 \mathrm{~cm}^{-1}$ of PI film by VDP in 
Figure 1 is sharper than that of $\mathrm{KBr}$ pellet. For the accuracy of the orientation analysis, the evaluation of the peak intensity from peak area may be, if anything, reasonable, but is inconvenient.

Any how, once enough attention is paid on the analytical procedure, this method can provide a convenient and powerful way for orientational characterization of organic thin films, and will be helpful in the understanding the relationship between the electrical properties and molecular orientation of the thin films.

Acknowledgment. We are grateful to Prof. T. Kato for helpful discussions.

\section{REFERENCES}

1. G. L. Wilkes, J. Macromol. Sci., Revs. Macromol. Chem., C10, 149 (1974).

2. R. S. Stein, Rubber Chem. Tech., 49, 458 (1976).

3. I. M. Ward, Adv. Polym. Sci., 66, 81 (1985).

4. D. L. Allara and R. G. Nuzzo, Langmuir, 1, 52 (1985).

5. J. Umemura, T. Kamata, T. Kawai, and T. Takenaka, J. Phys.
Chem., 94, 62 (1990).

6. L. G. Kazaryan, D. Ya. Tsvankin, B. M. Ginzburg, Sh. Tuichiev, L. N. Korzhavin, and S. Ya. Frenkel, Polym. Sci. USSR, 14, 1344 (1972).

7. S. Isoda, H. Shimada, M. Kochi, and H. Kambe, J. Polym. Sci., Polym. Phys. Ed., 19, 1293 (1981).

8. N. Takahashi, D. Y. Yoon, and W. Parrish, Macromoelcules, 17, 2583 (1984).

9. T. P. Russell, H. R. Brown, and D. T. Grubb, J. Polym. Sci. B, Polym. Phys., 25, 1129 (1987)

10. T. P. Russell, H. Gugger, and S. D. Swalen, J. Polym. Sci., Polym. Phys. Ed., 21, 1745 (1983).

11. M. A. Perez, Y. Ren, R. J. Farris, and S. L. Hsu, Macromolecules, 27, 6740 (1994).

12. K. Iida, T. Nohara, K. Totani, S. Nakamura, and G. Sawa, Jpn. J. Appl. Phys., 28, 2552 (1989).

13. J. L. Koenig, S. W. Cornell, and D. E. Witenhafer, J. Polym. Sci. $A-2, \mathbf{5}, 301$ (1967).

14. H. Ishida, S. T. Wellinghoff, E. Baer, and J. L. Koenig, Macromolecules, 13, 826 (1980).

15. J. H. Jou and P. T. Huang, Macromolecules, 24, 3796 (1991).

16. T. Strunskus, C. Hahn, D. Frenkel, and M. Grunze, J. Vac. Sci. Technol. A, 9, 1272 (1991).

17. M. Grunze, W. N. Unertl, S. Gnanarajan, and J. French, Mater. Res. Soc. Symp. Proc., 108, 189 (1988). 